\title{
A NEW TECHNIQUE FOR ACCURATELY DEFINED DEPOSITION OF CATALYST THIN FILMS IN DEEP FLOW CHANNELS OF. HIGH-TEMPERATURE GAS MICROREACTORS
}

\author{
R.M. Tiggelaar ${ }^{1,},{ }^{*}$, J.W. Berenschot ${ }^{1}$, R.E. Oosterbroek ${ }^{1}$, P. van Male ${ }^{2}$, \\ M.H.J.M. de Croon ${ }^{2}$, J.C. Schouten ${ }^{2}$, A. van den Berg ${ }^{1}$ and M.C. Elwenspoek ${ }^{1}$ \\ ${ }^{1} \mathrm{MESA}^{+}$Research Institute, University of Twente, Enschede, The Netherlands \\ ${ }^{2}$ Laboratory of Chemical Reactor Engineering, Eindhoven University of Technology, Eindhoven, The Netherlands \\ * eniail; r.m.tiggelaar@utwente.nl
}

\begin{abstract}
By using microreactors fabricated with silicon microtechnology, heterogeneous catalyzed gas-phase reactions can be studied which are difficult to control because of their exothermic nature, are explosive or use toxic/hazardous gases. In this type of microreactors, catalytic materials like rhodium or platinum are deposited on a thin membrane in deep trenches.

Conventional techniques, like lift-off lithography, cannot be used in deep trenches and deposition through flat shadow masks does not yield well-defined regions of catalyst. For well-controlled deposition of a catalytic thin film on a membrane located in a deep trench, a technique is developed using sputter deposition with a 3dimensionally shaped 'self-aligning' shadow mask.
\end{abstract}

\section{INTRODUCTION}

Due to the small dimensions of the gas flow channels in silicon-technology based microreactors (high-surface-tovolume ratios) and integration of functional elements like heaters and sensors, it becomes possible to control hightemperature catalytic partial oxidation reactions and study their reaction kinetics [1]. In this study, a microreactor is designed and fabricated for research on the Rh-catalyzed direct catalytic partial oxidation (CPO) of methane into synthesis gas. Synthesis gas is one of the most important intermediate products in the chemical industry.

Rh-catalyzed direct CPO starts when a gas mixture of methane and oxygen is heated up to $600{ }^{\circ} \mathrm{C}$ : The exothermic CPO reaction causes the temperature to go up as high as $1000^{\circ} \mathrm{C}$ [2]. Initial heating of the gas mixture takes place with Pt heating filaments, located on a thin membrane (figure 1). In order to guarantee good mechanical stability of the membrane at high temperatures as well as efficient heat transfer, the membrane is made of heavily boron-doped silicon and a thin electrical isolation layer of low stress silicon nitridè.

\section{CATALYTIC PARTIAL OXIDATION OF METHANE}

The direct catalytic partial oxidation of methane into synthesis gas ideally occurs as described by the following exothermic reaction (1):

$$
2 \mathrm{CH}_{4}+\mathrm{O}_{2} \rightarrow 2 \mathrm{CO}+4 \mathrm{H}_{2}
$$

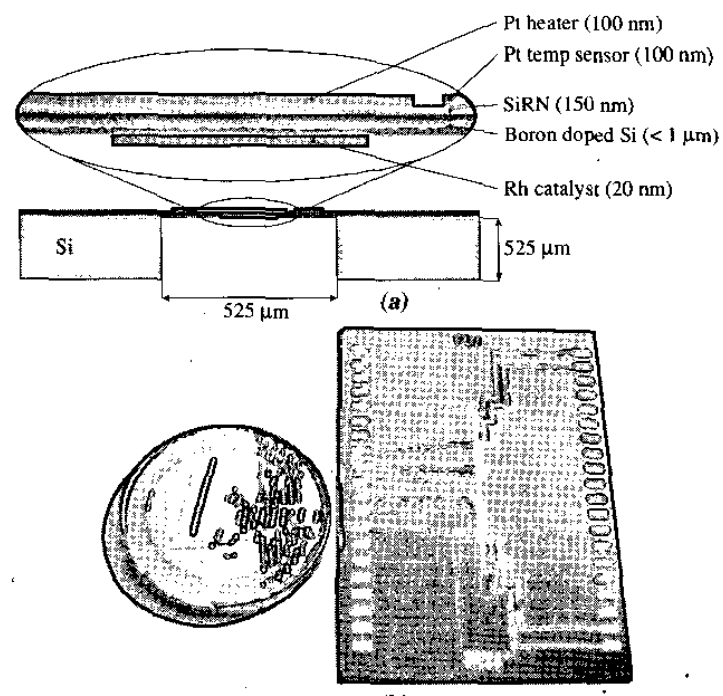

(b)

Figure 1: Cross-section of flow channel (only top wafer) (a) \& photo of microreactor $\left(3.0 \times 4.5 \times 0.1 \mathrm{~cm}^{3}\right)(b)$

However, the selectivities towards carbon monoxide (CO) and hydrogen $\left(\mathrm{H}_{2}\right)$ can be influenced by the simultaneous occurrence of 3 other reactions, viz. total combustion of methane, and secondary reactions of car-bon monoxide and hydrogen. All these three reactions result in (unwanted) formation of carbon dioxide $\left(\mathrm{CO}_{2}\right)$ and/or water $\left(\mathrm{H}_{2} \mathrm{O}\right)$ [3]. Furthermore, these 3 highly exothermic, non-selective oxidation reactions can result "in high catalyst temperatures which have negative influences on the stability of the catalyst and may cause heat-transport limitations.

Thermodynamics indicate that at low temperatures, atmospheric pressure and $2: 1 \quad \mathrm{CH}_{4} / \mathrm{O}_{2}$ ratio the equilibrium product composition consists of a mixture of total and partial oxidation products. At higher temperatures, however, synthesis gas can be produced with $100 \%$ selectivity [4].

In order to prevent formation of secondary products (water and carbon dioxide) it is important to apply the catalytic material only on the hottest part (the middle) of the membrane of the microreactor (figure 1(a)).

TRANSDUCERS ' 03

The 12th International Conference on Solid State Sensors, Actuators and Microsysterns, Boston, June 8-12, 2003 
Catalyst materials often used for CPO reactions and thoroughly analyzed are group VIII metals (Fe, $\mathrm{Co}, \mathrm{Ni}$, $\mathrm{Ru}, \mathrm{Rh}, \mathrm{Pd}, \mathrm{Ir}, \mathrm{Re}$, and $\mathrm{Pt}$ ) [5]. In general, nickel (Ni), platinum $(\mathrm{Pt})$ and rhodium $(\mathrm{Rh})$ have been identified as being most suitable as catalysts for direct catalytic partial oxidation in terms of selectivity and conversion [3].

For direct CPO of methane into synthesis gas, rhodium is the best choice. Rhodium shows a good catalytic activity as well as a high stability of the catalyst over a broad temperature range. Nickel has the disadvantage that its catalytic activity decreases at higher tempe-ratures due to oxidation, volatilization (metal evapora-tion) and, to a lesser extent, carbon deposition. Platinum as a catalyst also doesn't exhibit a stable performance at elevated temperatures:- at catalyst temperatures above $1000{ }^{\circ} \mathrm{C}$ conversions of methane and oxygen and selectivities towards hydrogen and carbon monoxide change due to the formation of volatile Pt oxide species. Deposition of these Pt oxide species on 'cold walls' of a CPO-system may result in secondary reactions of carbon monoxide and hydrogen, which results in a change in selectivity towards synthesis gas.

Based on the fact that the microreactor (figure 1) is to be used for research on the direct CPO of methane into synthesis gas, it was decided to deposit rhodium as catalyst material in the flow channel of the micro-reactor.

DEPOSITION OF METALS IN RECESSED AREAS

As shown in figure 1(a), a thin film of rhodium has to be deposited in the middle of the flow channel. This flow channel is a trench with a width and a depth of $525 \mu \mathrm{m}$. The metal film is deposited on a thin, fragile membrane $(<$ $1 \mu \mathrm{m}$ ) for optimal thermal contact to the catalyst. The width of the catalyst film was chosen to be $125 \mu \mathrm{m}$.

In general there are two methods which can be used to deposit a metal thin film recessed areas in silicon:

\section{Photoresist on non-planar silicon surfaces}

Conventional lithography and (ultrasonic) lift-off cannot be used in recessed areas, since it is difficult to obtain a conformal resist layer over a non-planar silicon surface by standard resist techniques (spinning, spraying or dipping). The resist layer will be very thin on convex corners, very thick a concave corners (e.g. in the bottom of a trench) and may be discontinuous. This results in a poor lithographic resolution.

It is, however, possible to obtain a conformal resist coating on non-planar silicon surfaces (3D-surfaces) by using electrodepositable photoresist [6]: After electrodeposition of resist in anisotropically etched cavities in (100)-silicon, the step coverage on the $54.7^{\circ}$ inclined sidewalls ( 111$\}$-planes) was excellent and lines with widths down to $20 \mu \mathrm{m}$ could be patterned in cavities (depths up to $200 \mu \mathrm{m}$ ) without problems. Recently, new photoresist coating methods for 3D structured wafers have been developed. Kutchoukov et. al. obtained a uniform spin-coated resist layer on structured (100)-silicon (anisotropically etched holes and trenches) after smoothing convex and concave corners using a short etch step in TMAH [7]. This smoothening resulted in conformal spin-coated resist layers on $54.7^{\circ}$ sloped sidewalls and the resist patterning resolution was high. Subsequent deposition of metals over the sidewalls of recessed areas up to $450 \mu \mathrm{m}$ was done successfully (metal line widths down to $100 \mu \mathrm{m}$ ).

\section{Shadow masks of silicon}

Another method for deposition of metals in deep trenches and/or non-planar substrate surfaces is direct deposition through shadow masks made of silicon.

Conventional shadow masks have a planar (flat) surface. This surface contains holes and trenches which form the pattern/structure to be transferred to a substrate. If evaporation of a metal is used in combination with a conventional planar shadow mask, metal patterns with a resolution of $3 \mu \mathrm{m}$ in $380 \mu \mathrm{m}$ deep holes could be obtained if a short etch dip with an appropriate etchant is carried out in order to remove spreaded atoms [8]. Spreading is a disadvantage of planar shadow masks: if the recessed areas of the substrate in which metal is to be deposited is deep, the large distance between the maskopenings (apertures) and the substrate may cause resolution problems due to spreading caused by scattered vapor atoms.

The use of a non-planar shadow mask overcomes the mentioned resolution limits. A 3D-shadow mask can be designed such that it fits exactly in the non-planar substrate on which the patterns are to be deposited. By micromachining processing, sections of a silicon shadow mask can be removed such that it matches the topography of the substrate: the 3D-shadow mask is a negative replica of the substrate [9]. Using this method, the maskopenings/apertures can be positioned very close to the substrate such that resolution is improved. Furthermore, when a 3D-shadow mask is fabricated in (100)-silicon using $\mathrm{KOH}$-etching, $54.7^{\circ}$ inclined $\{111\}$ planes can be used for self-alignment features of the shadow mask with respect to the device wafer. With the 'self-aligning' 3Dshadow mask, the definition of evaporated or sputtered metal films is improved drastically, as well as the resolution of lithographically exposed patterns in recessed areas with large sloped steps. It was possible to evaporate very well-defined $10 \times 50 \mu \mathrm{m}^{2}$ metal spots in a cavity of $525 \mu \mathrm{m}$ when the gap between the mask-opening and substrate was $70 \mu \mathrm{m}$ [9]. The edge-definition of the deposited pattern can be further improved by reducing the gap, even down to zero for deposition on non-fragile surfaces. The self-aligning feature on $54.7^{\circ}$ sloped sidewalls can also be used for defining metal wires across these sloped steps: Brugger et. al. [9] showed that aluminum wires of $25 \times 0.5 \mu \mathrm{m}^{2}$ could be evaporated across a $120 \mu \mathrm{m}$ high step without loss of dimension control or pattern. 
The flow channel of the presented microreactor has a rectangular cross-section. Hence, the sidewalls of the flow channel are vertical. This implies that using photoresist for patterning a Rh-track on the bottom of the flow channel is not possible (an advantage is though that metal deposition on vertical sidewalls will be less, however this will depend on the deposition method used). A second reason why resist cannot be used is that the Rh-catalyst is to be deposited on a very fragile membrane. Use of resist (spinning, lithopgraphy, deposition and subsequent ultrasonic stimulation of the lift-off procedure); will dramatically increase the risk of fatal membrane damage. Finally, the influence of chemicals involved in the photolithographic process on the catalytic activity of rhodium are unknown. In conclusion, the use of a shadow mask is highly favored:

As mentioned earlier, deposition of catalyst material on the cold sidewalls of the flow channel has to be avoided. Hence a 3D-shadow mask needs to be applied. However, accurately defined deposition of thin film layers in deep trenches $(\geq 400 \mu \mathrm{m}$ - width of trench $\sim 525 \mu \mathrm{m})$ without sidewall deposition, a thin film width of $>100 \mu \mathrm{m}$, centered on fragile, thin membranes and gaps (distance between mask-opening and membrane) of $75 \mu \mathrm{m}$ or smaller is impossible with shadow masks made of (100)silicon. This challenging technological problem is concurred by fabricating a shadow mask in (110)oriented silicon.

\section{DESIGN AND FABRICATION OF A 3D, 'SELF- ALIGNING SHADOW MASK OF (110)-SILICON} The requirements summarized in the above paragraph are fulfilled by fabricating a 3-dimensional, high-aspect ratio 'self-aligning' shadow mask, using $\mathrm{KOH}$-etching of a 1 $\mathrm{mm}$ thick (110)-oriented silicon wafer. Figure 2 shows a schematic view of this mask.

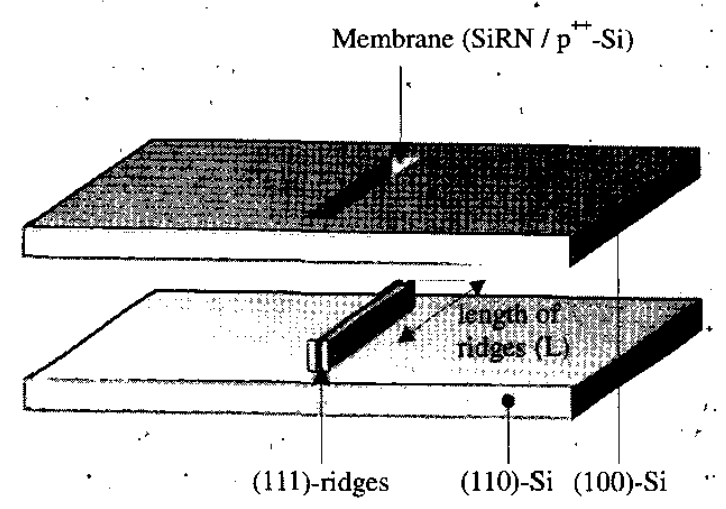

Figure 2: 3D-representation of 'self-aligning' shadow mask

The crystallographic orientation of (110)-oriented silicon allows the fabrication of extremely narrow grooves, having a width-to-depth aspect ratio up to $100: 1$, when (110)-silicon is etched in potassium hydroxide $(\mathrm{KOH})$ [10]. $\{111\}$-planes which have an angle of $90^{\circ}$ with respect to the wafer surface can be revealed. These vertical planes can be used to create alignment-ridges which can be lowered into the flow channel of the microreactor. Between these alignment ridges a slit is created through which the rhodium can be sputtered on the membrane.

The length (L) of the ridges is almost identical to the length of the flow channel $(30 \mathrm{~mm})$ and therefore guarantees self-alignment of the shadow mask with respect to the flow channel of the microreactor. In figure 3 close-ups of the lowering of the shadow mask in the channel of the microreactor are shown, as well as dimensions of the shadow mask.

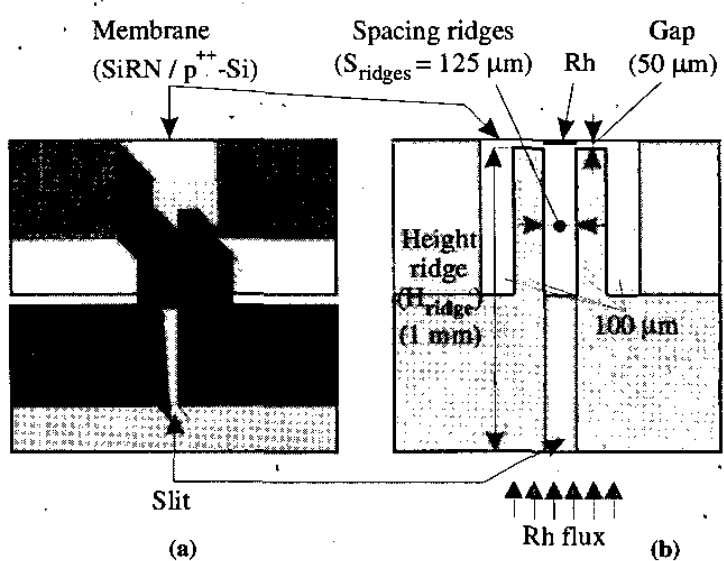

Figure 3: Schematic view of lowering 'self-aligning' mask in deep flow channel (a) \& sputtering after positioning the mask in the channel with capping membrane (b)

After fabrication of the mask, a slit (opening through the silicon) with a width of $125 \mu \mathrm{m}$ and a height of $1 \mathrm{~mm}$ is obtained. This slit defines the catalyst pattern which is sputtered on the membrane. The gap between the ridges and the membrane is about $50 \mu \mathrm{m}$, to ensure no direct contact between the 'ridges and the fragile membrane. However, smaller gaps, down to $10 \mu \mathrm{m}$ can be obtained. The smaller the gap, the better the resolution of the deposit (less spreading). Finally; the aspect ratio of ridge height versus spacing between the ridges (slit width), $\mathrm{H}_{\text {ridge }} / \mathrm{S}_{\text {ridges }}$, is $8(1 \mathrm{~mm} / 125 \mu \mathrm{m})$, which is high, but in theory ratios up to 50 are feasible.

The fabrication of the shadow mask is done using a $1 \mathrm{~mm}$ thick (110)-oriented silicon wafer. The thickness of $1 \mathrm{~mm}$ is selected for stability and handling purposes during fabrication and sputtering: the shadow mask must be about twice as thick as the depth of the flow channel. The fabrication process is given in figure 4.

First a layer of $200 \mathrm{~nm}$ low stress silicon nitride is deposited directly. on a (110)-silicon wafer (A). Then, the exact crystallographic orientation of the wafer is determined using an alignment mask [11]. Next, the slit is defined at the backside of the wafer using lithography. After plasma etching of SiRN the slit is etched down to a depth of $100 \mu \mathrm{m}$ (B). The second lithographic step defines the alignment ridges on the topside of the silicon. : 


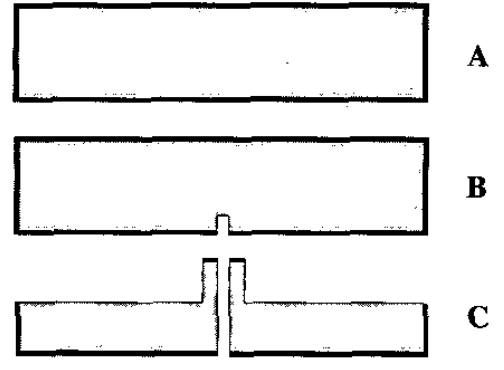

Figure 4: Schematic presentation of the fabrication process of the 3D high-aspect ratio 'self-aligning' shadow mask

Plasma etching is used to remove the SiRN on the topside. Subsequently, double sided $\mathrm{KOH}$-etching (top and back side of the wafer are etched in $25 \mathrm{wt} \% \mathrm{KOH}$ at $75^{\circ} \mathrm{C}$ ) is used to create the alignment ridges and slit (C). Etching the wafer $450 \mu \mathrm{m}$ from both sides results in a completely opened slit. Finally, the SiRN is removed by etching in $50 \% \mathrm{HF}$

\section{RESULTS}

Sputter deposition of different catalysts (rhodium and platinum) and tantalum, which is often used as adhesion layer, was successfully carried out using the 3D highaspect ratio 'self-aligning' shadow mask. Figure 5 shows a topview of a Rh-catalyst track underneath the capping membrane of a microreactor which can be used for $\mathrm{Rh}$ catalyzed CPO of methane.

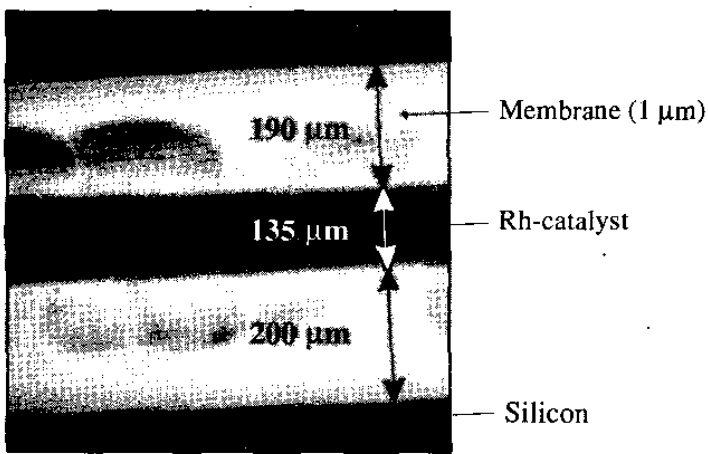

Figure 5: Picture (top view) of Rh-catalyst undemeath the thin capping membrane of the microreactor

The sputtered films turned out to be slightly wider than the width of the slit. The slit width was $125 \mu \mathrm{m}$, whereas the deposited rhodium track was 135-140 $\mu \mathrm{m}$. The difference is due to spreading of the sputtered atoms: the gap of $50 \mu \mathrm{m}$ between the membrane and the alignment ridges allows some spreading of the atoms. This resolution of the track can be improved by using a shadow mask with higher alignment ridges (viz. a smaller gap) or by using more directional deposition methods than sputtering, e.g. evaporation. Furthermore, no deposit was found on the sidewalls of the flow channel, such that the aim of avoiding catalyst deposition on 'cold' sidewall surfaces of the flow channel was reached. Finally, it is observed that the deposition rate of rhodium through the shadow mask is a factor of 10 lower than its bulk deposition rate. For $\mathrm{Ta}$ and $\mathrm{Pt}$ the deposition rates equal their bulk sputter deposition rates. This phenomenon is currently investigated in detail.

\section{CONCLUSIONS}

A technique for well-controlled deposition of catalyst material underneath the capping membrane of silicon microreactors has been developed. A shadow mask, designed and fabricated in (110)-oriented silicon can be used to deposit accurately defined catalyst patterns in deep flow channels with vertical sidewalls. By using sputter deposition with the 3D shaped high-aspect ratio 'self-aligning' shadow mask, it is possible to create metal tracks of $135 \mu \mathrm{m}$ in a rectangular flow channel of $525 \times 525 \mu \mathrm{m}^{2}$. Sidewall deposition of catalyst material, which could lead to unwanted secondary reactions, is indeed avoided.

With this technique, well-defined films of a Rh-catalyst were deposited in the flow channel of a microreactor which is used for studying Rh-catalyzed direct CPO of methane.

\section{ACKNOWLEGDGEMENTS}

This work is supported by the Dutch Technology Foundation (STW - project 'FORSiM', nr. EFC.5134).

\section{LITERATURE}

[1]: R. Srinivasan et. al.: Micromachined reactor for catalytic partial oxidation reactions - AIChE J., 43 (11), 3059 (1997).

[2]: D.A. Hickman et. al.: Synthesis gas formation by direct oxidation of methane over Rh monoliths - Catal. Lett., 17, 223 (1993).

[3]: C.R.H. de Smet: Partial oxidation of methane to synthesis gas: reaction kinetics and reactor modelling, $\mathrm{PhD}$-thesis Eindhoven University of Technology, Eindhoven, The Netherlands (2000).

[4]: E.P.J: Mallens: A reaction path analysis of the catalytic partial oxidation of methane by transient experiments, PhDthesis - Eindhoven University of Technology, Eindhoven, The Netherlands (1996).

[5]: P.M. Torniainen et. al.: Comparison of monolith-supported metals for the direct oxidation of methane to syngas - J. Catal., 146, 1 (1994).

[6]: P. Kersten et. al.: Photolithography on micro-machined 3D surfaces using electrodeposited photoresists - Sens. \& Act. A, 51, 51 (1995).

[7]: V.G. Kutchoukov et. al.: New photoresist coating method for 3-D structured wafers - Sens. \& Act. A, 85, 377 (2000).

[8]: G.J. Burger et. al.: High-resolution shadow-mask patterning in deep holes and its application to an electrical wafer feedthrough - Sens. \& Act. A, 54, 669 (1996).

[9]: J. Brugger et. al.: Self-aligned 3D shadow mask technique for patterning deeply recessed surfaces of micro-electromechanical systems devices; Sens \& Act A, 76, 329 (1999).

[10]:L.D. Kendall: Vertical etching of silicon at very high aspect ratios - Ann. Rev. Mater. Sci., 9, 373 (1979).

[11]:M. Vangbo et. al.: Precise mask alignment to the crystallographic orientation of silicon wafers using wet anisotropic etching - J.' Micromech. Microeng., 6, 279 (1996). 